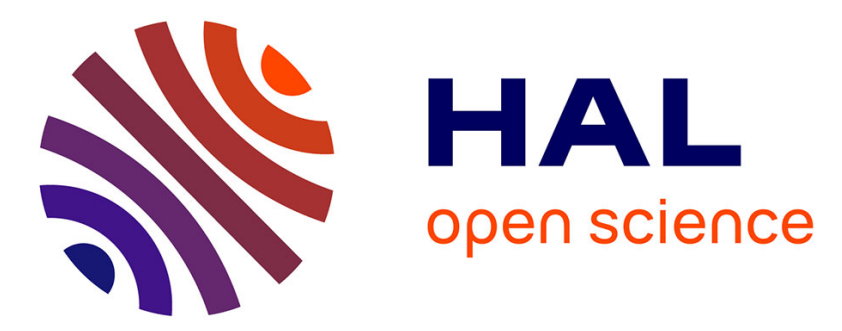

\title{
Terahertz near-field imaging using a Y splitter and Sommerfeld wire waves on bare metal rods
}

Jean-Paul Guillet, Laurent Chusseau, R. Adam, T. Laurent, T. Grosjean, D. Charraut, A. Pénarier

\section{To cite this version:}

Jean-Paul Guillet, Laurent Chusseau, R. Adam, T. Laurent, T. Grosjean, et al.. Terahertz near-field imaging using a Y splitter and Sommerfeld wire waves on bare metal rods. 35th International Conference on Infrared, Millimeter and Terahertz Waves, Sep 2010, Rome, Italy. 10.1109/ICIMW.2010.5612583 . hal-00584459

\section{HAL Id: hal-00584459 \\ https://hal.science/hal-00584459}

Submitted on 15 Apr 2021

HAL is a multi-disciplinary open access archive for the deposit and dissemination of scientific research documents, whether they are published or not. The documents may come from teaching and research institutions in France or abroad, or from public or private research centers.
L'archive ouverte pluridisciplinaire HAL, est destinée au dépôt et à la diffusion de documents scientifiques de niveau recherche, publiés ou non, émanant des établissements d'enseignement et de recherche français ou étrangers, des laboratoires publics ou privés.

\section{(c)(1)}

Distributed under a Creative Commons Attribution| 4.0 International License 


\title{
Terahertz near-field imaging using a Y splitter and Sommerfeld wire waves on bare metal rods
}

\author{
J.-P. Guillet ${ }^{\mathrm{a}}$, L. Chusseau ${ }^{\mathrm{a}}$, R. Adam ${ }^{\mathrm{a}}$, T. Laurent ${ }^{\mathrm{a}}$, T. Grosjean ${ }^{\mathrm{b}}$, A. Penarier ${ }^{\mathrm{a}}$ and D. Charraut ${ }^{\mathrm{b}}$ \\ ${ }^{a}$ IES, UMR 5214 CNRS, Université Montpellier 2, 34095 Montpellier, France \\ ${ }^{\mathrm{b}}$ FEMTO-ST, UMR 6174 CNRS, Université de Franche Comté, 25030 Besançon, France
}

\begin{abstract}
We describe a near-field experiment using a Y splitter supporting cw THz Sommerfeld wire modes coupled and decoupled with differential phase plates. Owing to a taper up to $\sim 50$ $\mu \mathrm{m}$ of one of the $\mathrm{Y}$ ends, a near-field imaging resolution of $\sim 90$ $\mu \mathrm{m}$ is demonstrated in agreement with calculations.
\end{abstract}

\section{INTRODUCTION AND BACKGROUND}

$\mathrm{N}_{\text {ton }}$ EAR-FIELD imaging is a key technique for high resolution imaging. This is especially valuable in the terahertz $(\mathrm{THz})$ range where the very long wavelength has a disastrous influence of the far-field imaging resolution limit. Besides the revamping of scanning near-field optical microscopy in the $\mathrm{THz}$ range, original systems taking benefit from Sommerfeld wire waves on bare metal $\operatorname{rods}^{1}$ have been recently developed $^{2,3}$ since such waves have been demonstrated to be very efficient and easy to manage at $\mathrm{THz}$ frequencies ${ }^{4-6}$.

Aiming at developing $\mathrm{cw}$ near-field imaging with versatile $\mathrm{THz}$ sources we already showed the combination of Sommerfeld wire modes excitation with a differential phase element and the longitudinal field selectivity of a tapered needle as near-field probe ${ }^{3}$. Awad et al. $^{2}$ showed separately that such a needle may provide large sub-wavelength resolution up to the $\mu \mathrm{m}$ scale. In this work, we carefully analyze the propagation of Sommerfeld waves on stainless steel rods. By combining them in a Y splitter ${ }^{4,6}$ with a needle at one end and two differential phase plate elements, we obtain a cw near-field excitation near-field detection setup in the $\mathrm{THz}$ range. A great advantage of this setup is the coupling with external sources and detectors that involves only linearly polarized plane waves.

\section{METHOD AND RESUltS}

The proposed $\mathrm{THz}$ microscope system is shown in Fig. 1. It involves the coupling and propagation of Sommerfled waves along metallic wires. We already showed such coupling and propagation using a set of two parabolic mirrors and a Teflon differential phase element (DPE) ${ }^{3}$. The DPE induces a $\pi$-phase difference on half the input beam cross-section so that it efficiently overlaps with the radially polarized wire mode.

Sommerfled wave excitation is obtained with a microwave synthesizer followed by a $75-110 \mathrm{GHz}$ sextupler electronic source from Spacek Labs Inc delivering $\sim 0 \mathrm{dBm}$ to a rectangular horn antenna. The linearly-polarized beam is focused and transformed into Sommerfled waves using two off-axis parabolic mirrors and a DPE. The detection is ensured by a similar quasi-optical system in front of the rectangular horn antenna coupled to a Schottky diode and a microwave spectrum analyzer. With acceptable settings of the spectrum analyzer, the noise detection level of the overall detection is estimated to $-80 \mathrm{dBm}$. Stainless steel wires of $0.8 \mathrm{~mm}$ diameter and of known bulk resistivity $1.3210^{6} \Omega^{-1} \mathrm{~m}^{-1}$ are placed in-between the two DPE couplers of diameter $6 \mathrm{~cm}$. DPEs also serve as wire holders eventually supplemented by a Teflon disk of same diameter if long wires are considered. Emission and detection systems, including DPE and horn antennas, are crosspolarized to reduce any direct coupling. Additionally, an absorbing diaphragm with a clear aperture of $3 \mathrm{~cm}$ is used to cancel any direct transmission between horns while keeping a theoretical transmission in excess of $90 \%$ for the Sommerfeld wave. This was verified by measurements without the wire.

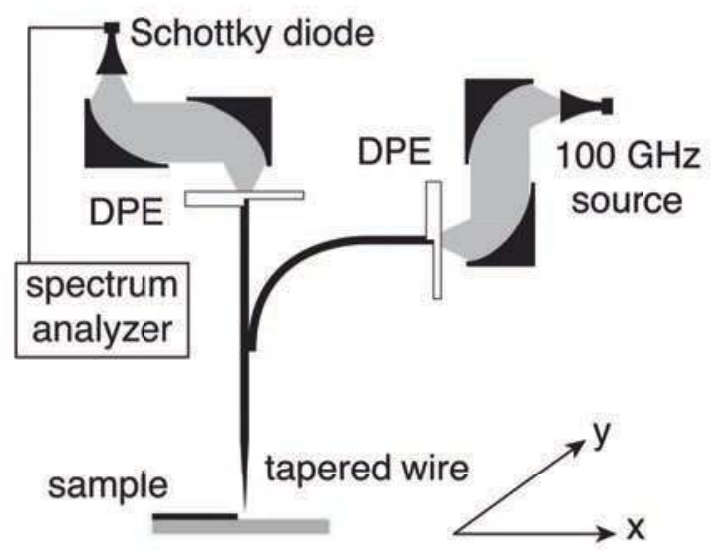

Fig. 1: Near-field experimental setup.

To optimize the system design, we conducted preliminary experiments to evaluate the various source of losses. Using successive cutback experiments, propagation losses of 0.2 $\mathrm{dB} / \mathrm{cm}$ are measured, a value 5 times greater than the 0.04 $\mathrm{dB} / \mathrm{cm}$ calculated ${ }^{1}$, perhaps because of wire surface oxidation or contamination with organic materials that might have induced excess losses in combination with the low $\sim 1 \mu \mathrm{m}$ skin depth. Another result of this experiment was the estimation of a 32\% DPE coupling efficiency deduced from the extrapolated transmission at null wire length as compared to the direct coupling between horns. This coupling efficiency is very close to the $\sim 35 \%$ calculated value that we obtained from the known theoretical field profiles ${ }^{1}$ and for an input Gaussian beam of waist $\sim 11 \mathrm{~mm}$.

In a second experiment, the radial extension of the wire mode was estimated using an iris around the rod that reduces progressively the allowed clear space for the wire mode transmission. We measured the power transmission by the Sommerfeld waves while the clear aperture of the iris is var- 
ied. As shown in Fig. 2, the propagating mode is confined in a cylinder volume of radius about 5 times the wire radius $(0.8 \mathrm{~mm}$ in our case). Notice that results at $220 \mathrm{GHz}$ are very noisy because our detection system is very inefficient in that case. We calculated the corresponding transmission using the theoretical field profiles ${ }^{1}$ and obtained a nearly perfect comparison with experiments, the mode being more confined at higher frequency. Nonetheless calculations show that the mode concentration in a cylinder volume of $\sim 5$ times the wire radius scales down up to the $\mu \mathrm{m}$ size, thus allowing a high field concentration at the near-field probe. $^{7}$
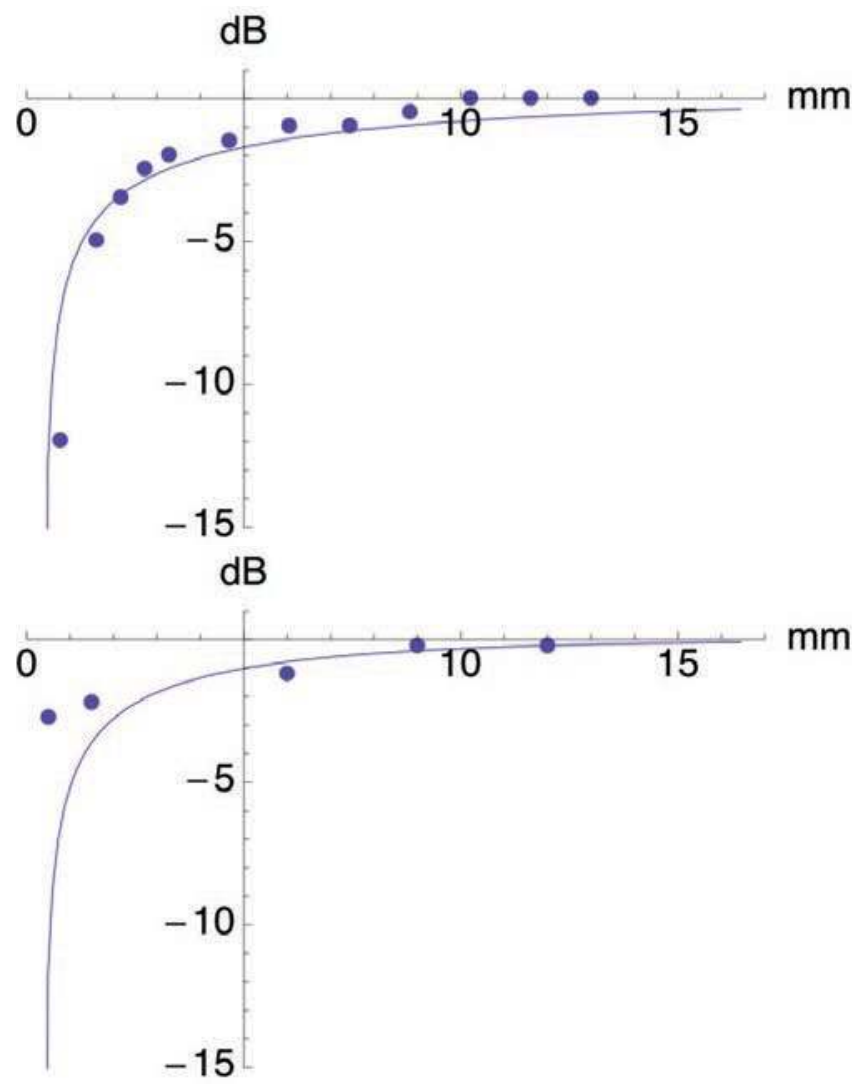

Fig. 2: Power transmissions of a Sommerfeld wire mode through an iris of variable clear aperture (abcissa is radius in $\mathrm{mm}$ ). Upper curve: $100 \mathrm{GHz}$, lower curve: $220 \mathrm{GHz}$. Dots: measurement. Solid line: calculations.

Bending losses were evaluated with the same setup. We used the natural flexibility of metals by increasing the angle between emission and detection to progressively bend the wires. Figure 3 reports the measured losses per unit length of two wires with different metal nature and diameters. This procedure is different from that used in previous reports of the same measurement ${ }^{6,8}$, but it produces very similar results as seen in Fig. 3. Note that bending radius below $15 \mathrm{~cm}$ are unattainable without inducing permanent distortion of the wire and large radius $\geq 60 \mathrm{~cm}$ are also impossible because they cannot be distinguished from the natural bending of the wire induced by its own weight. Also worth to be pointed out are the losses that appear nearly metal independent. This was previously suggested $^{6}$ and also validated by our own calculations. As a result, the differences between the two experimental curves are most likely due to the different wire radius than to the metal nature.

In the microscope of Fig. 1, we found necessary to introduce a right angle between source and detector to cancel the direct coupling due to sample specular reflection. It results in a $90^{\circ}$ bend of $20 \mathrm{~cm}$ radius on one wire that induces a $\sim 30 \mathrm{~dB}$ attenuation. Therefore, even with a perfect Y-splitter and an adiabatic focusing needle, large attenuations take action during image acquisition and reduce the signal to noise ratio.

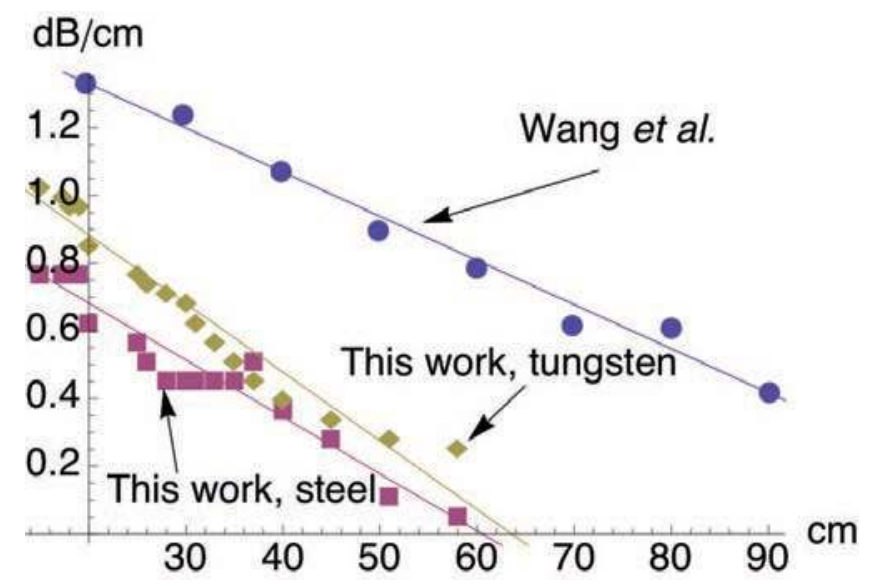

Fig. 3: Measured attenuations of Sommerfeld wire modes as a function of the radius of curvature. Two wires were tested at $100 \mathrm{GHz}$, a $0.8 \mathrm{~mm}$ diameter stainless steel and a $0.5 \mathrm{~mm}$ tungsten wire. Dots: measurement. Solid line: linear fits. Results are compared to Wang and Mittleman results of Ref. [6].

In a final integration, the $\mathrm{Y}$ splitter was built and we mechanically attached a $4 \mathrm{~cm}$ long metal needle to one of its end. Needle diameter was chosen to fit that of the wire and its end was tapered over a distance of $5 \mathrm{~mm}$ up to a spike with a $50 \mu \mathrm{m}$ radius of curvature. This sharp end thus operates as a near-field probe sensitive to the longitudinal electric field ${ }^{2,3}$. The sample is raster-scanned right beneath the needle apex using two motorized translation stages and the probe height is adjusted before the scan with a manual translation stage of micrometer resolution. No active height control is applied during scans.

The imaging properties and the resolution ability of our setup were tested by scanning one metal $\mathrm{Au}$ ) right angle corner deposited on glass substrate. For an estimated $\sim 10 \mu \mathrm{m}$ flyover distance, a 1D scan obtained with a $5 \mu \mathrm{m}$ linear step in given in Fig. 4. This scan exhibits a piecewise evolution with a linear transition from the upper level (probe over the metal) to the lower level (probe over the glass substrate). The transition between both occurs over a distance of $90 \mu \mathrm{m}(10 \%$ to $90 \%$ edge criterion). This value corresponds to $\lambda / 33$ and is close to the $50 \mu \mathrm{m}$ characteristic size of the needle apex. The measured varying signal then comes from the near-field and corresponds to the detection of the longitudinal electric-field component ${ }^{2,3}$. This was confirmed by calculating from the theoretical field profiles $^{1}$, a $660 \mu \mathrm{m}$ radial field extension at $-3 \mathrm{~dB}$ for a $50 \mu \mathrm{m}$ diameter stainless steel wire. As a consequence, any variation 
in the detected signal that may originate from the radial field component would have extended over more than the spans used in experiments and just produced an increase of the background.

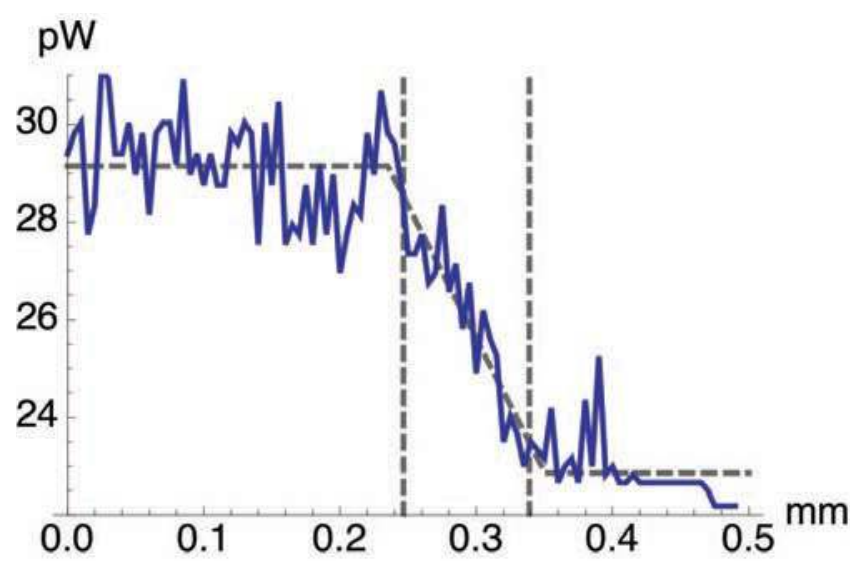

Fig. 4 : Measured transfer function during the probe flyover on the edge of an Au line on glass substrate. The dashed lines are the measurement piecewise approximations used for evaluating resolution.

\section{CONCLUSION}

We have built a versatile cw measurement setup for terahertz near-field reflectometry with a $\lambda / 33$ resolution. Measurement examples have shown a detected power of only $\sim 30 \mathrm{pW}$ (i.e. $-75 \mathrm{dBm}$ ) that is very close to the detection background level of our experimental setup. As characterized, most of the losses come from the wire bending. Changing the geometry in next evolutions of the setup can eventually reduce them efficiently. Improvements are also expected both in resolution and sensitivity by using sharper needles for which high electric field enhancements are expected in the near-field ${ }^{7}$.

\section{REFERENCES}

[1] G. Goubau, "Surface waves and their application to transmission lines," J. Appl. Phys., 21, pp. 1119-1128, 1950.

[2] M. Awad, M. Nagel, and H. Kurz, "Tapered Sommerfeld wire terahertz near-field imaging," Appl. Phys. Lett., 94, 051107, 2009.

[3] R. Adam, L. Chusseau, T. Grosjean, A. Penarier, J.-P. Guillet, and D. Charraut, "Near-field wire-based passive probe antenna for the selective detection of the longitudinal electric field at terahertz frequencies," J. Appl. Phys., 106, 073107, 2009.

[4] K. Wang and D. Mittleman, "Metal wires for terahertz wave guiding," Nature, 432, pp. 376-379, 2004.

[5] T. Jeon, J. Zhang, and D. Grischkowsky, "THz Sommerfeld wave propagation on a single metal wire," Appl. Phys. Lett., 86, 161904, 2005.

[6] K. Wang and D. Mittleman, "Guided propagation of terahertz pulses on metal wires," J. Opt. Soc. Am. B, 22, pp. 2001-2008, 2005.

[7] Y. B. Ji, E. S. Lee, J. S. Jang, and T.-I. Jeon, "Enhancement of the detection of THz Sommerfeld wave using a conical wire waveguide," Opt. Express, 16, pp. 271-278, 2008.

[8] V. Astley, J. Scheiman, R. Mendis, and D. M. Mittleman, "Bending and coupling losses in terahertz wire waveguides," Opt. Lett., 35, pp. 553$555,2010$. 